\title{
Laboreal
}

Volume $17 \mathrm{~N}^{\circ} 1$ | 2021

Trabalhar hoje: mudanças, permanências, estratégias, reinvenções

\section{1 de marzo de 2020: el trabajo en la Atención Primaria de Salud (APS) interpelado por la pandemia de Covid-19}

11 de março de 2020: o trabalho na Atenção Primária à Saúde (APS) interpelado pela pandemia da Covid-19

11 mars 2020: les travaux dans les soins de santé primaires (SSP) interpeller par la pandémie Covid-19

March 11, 2020: work in Primary Health Care (PHC) challenged by the Covid-19 pandemic

\section{Ana Cláudia Barbosa da Silva-Roosli}

Traductor. Fernanda Romero (fernandaromero.trad@gmail.com)

\section{(2) OpenEdition}

\section{Journals}

Edición electrónica

URL: https://journals.openedition.org/laboreal/17703

DOI: $10.4000 /$ laboreal. 17703

ISSN: 1646-5237

Este artículo es una traducción de:

11 de março de 2020: o trabalho na Atenção Primária à Saúde (APS) interpelado pela pandemia da

Covid-19 - URL : https://journals.openedition.org/laboreal/17693 [pt]

Editor

Universidade do Porto

Referencia electrónica

Ana Cláudia Barbosa da Silva-Roosli, «11 de marzo de 2020: el trabajo en la Atención Primaria de Salud (APS) interpelado por la pandemia de Covid-19», Laboreal [En línea], Volume 17 No1 | 2021 , Publicado el 18 juin 2021, consultado el 28 juin 2022. URL: http://journals.openedition.org/laboreal/ 17703 ; DOI: https://doi.org/10.4000/laboreal.17703

Este documento fue generado automáticamente el 20 junio 2021.

Laboreal está licenciado com uma Licença Creative Commons - Atribuição-NãoComercial 4.0 Internacional. 


\title{
11 de marzo de 2020: el trabajo en la Atención Primaria de Salud (APS) interpelado por la pandemia de Covid-19
}

\author{
11 de março de 2020: o trabalho na Atenção Primária à Saúde (APS) interpelado \\ pela pandemia da Covid-19 \\ 11 mars 2020: les travaux dans les soins de santé primaires (SSP) interpeller par \\ la pandémie Covid-19 \\ March 11, 2020: work in Primary Health Care (PHC) challenged by the Covid-19 \\ pandemic
}

\section{Ana Cláudia Barbosa da Silva-Roosli}

Tradución : Fernanda Romero (fernandaromero.trad@gmail.com)

\section{Introducción}

Desde el 11 de marzo de 2020, con el reconocimiento de la pandemia de Covid-19 por parte de la Organización Mundial de la Salud (OMS), se ha llevado a cabo un esfuerzo global para su contención y así detener el crecimiento de enfermos y muertes. A nivel del trabajo en la salud, la evolución del Covid-19 exige puntos de intervención diversificados para atender a enfermos con diferentes necesidades, estadios de infección y espectro de gravedad: desde el "seguimiento de los casos leves en aislamiento domiciliario, con orientaciones para el manejo de los síntomas y la identificación precoz de los señales de alarma, hasta la hospitalización en unidades de cuidados intensivos (UCI) y la rehabilitación tras el alta hospitalaria" (Daumas et al., 2020, p. 1).

De manera desigual, esta diversidad puede ser abarcada por el sistema de salud brasileño, compuesto por una combinación entre el sistema público -el Sistema Único 
de Salud (SUS)- y un subsistema privado, orientado en su mayoría a la oferta de atención médico-hospitalaria (Teixeira et al., 2020). El SUS, creado en 1988 con el establecimiento constitucional de la salud como derecho, se guía por los principios de universalidad e integralidad.

Brasil es el único país del mundo con un sistema de salud pública universal para una población de más de 210 millones de habitantes (Guimarães, 2020). En la actualidad, 162 millones de personas dependen exclusivamente del SUS para cualquier tipo de atención. En el otro extremo, 47 millones de personas, con mayores rentas, adquieren voluntariamente planes de salud privados. Aun así, en algún momento, esta franja de la población también hace uso del SUS a través de acciones de vigilancia sanitaria, vacunación en masa o programas públicos de trasplante, por ejemplo (Dantas, 2020).

tasa de letalidad del Covid-19 llevó a Brasil, como a la mayoría de los países del mundo, a centrar su respuesta a la pandemia en el trabajo en salud operado en la atención hospitalaria, la gestión médica individual y el desarrollo de acciones curativas (Medina, Giovanella, Bousquat, Mendonça y Aquino, 2020). Aunque importante y necesaria, es posible enumerar varias limitaciones de esta directriz, empezando por la cobertura de camas hospitalarias en el país, que atiende sólo a $1 / 4$ de la población brasileña (Teixeira et al., 2020), con una concentración de más de $2 / 3$ de esta en el sistema de salud privado.

5 En este sentido, se hizo hincapié en el manejo clínico del nuevo coronavirus y en el aprendizaje de protocolos de medidas individuales (higiene y uso de equipos de protección) en detrimento de una discusión en torno a las condiciones necesarias para realizar el trabajo. Hay informes de los profesionales sobre las condiciones precarias, la higiene inadecuada, las jornadas de trabajo extenuantes, la falta de formación o la indisponibilidad o insuficiencia de equipos de protección, incluso en los servicios de cuidados intensivos (Jackson et al., 2020). Los índices entre los trabajadores de la salud parecen atestiguar esta realidad: hay más de 484 mil casos de enfermedad por Covid-19 (COFEN - Consejo Federal de Enfermería, 2021b) y al menos 990 muertes (Schmitt, 2021). Entre los profesionales de enfermería, el país responde por un tercio de las muertes mundiales por el virus (COFEN, 2021a).

6 La estrategia priorizada en Brasil negligencia otra dimensión del Covid-19: se trata de "una enfermedad comunitaria, que se dispersa muy rápidamente y se manifiesta de forma similar a otros síndromes gripales (SG), siendo de difícil contención" (Teixeira et al., 2020, p. 1), lo que hace cruciales las acciones de vigilancia de la salud con la realización de pruebas, la detección del mayor número de casos, el rastreo y el monitoreo de los contactos. La epidemióloga Fassa (2021, p. 19) es categórica: "un área en la que estamos cometiendo muchos errores es en la vigilancia" y defiende, junto con varios investigadores (Medina et al., 2021), su desarrollo y/o articulación con la Atención Primaria de Salud (APS) para minimizar los daños y reducir las muertes evitables.

7 Enfrentar la pandemia con el apoyo del trabajo en salud en el SUS exige una doble tarea: la superación de los límites de su condición real -dificultades en la gestión de sus demandas diarias, insuficiencia de la infraestructura de la red hospitalaria- y su reorganización en respuesta al marco sanitario instalado. 


\section{Las potencialidades del trabajo de la APS en la lucha contra la pandemia en Brasil}

8 La APS tiene una estructura amplia, con unidades de salud distribuidas por todo el país, llegando incluso a las regiones más desprovistas de otros recursos, lo que le otorga una cobertura estimada del $65 \%$ de la población nacional (Giovanella et al., 2021). Sus atribuciones prescritas orientan a que el trabajo se realice de manera territorializada, con acciones individuales y colectivas, contemplando la promoción, la prevención, el diagnóstico, el tratamiento, la rehabilitación y la reducción de daños.

9 En esta crisis, el trabajo en la APS puede dirigirse tanto a los ciudadanos no enfermos como a orientarse para la protección del contagio, la detección precoz de los pacientes sintomáticos, el rastreo de los contactos, el aislamiento, el tratamiento (Medina et al., 2020) y la rehabilitación de los pacientes con secuelas de Covid-19. También puede intervenir junto a grupos sociales vulnerables, identificar situaciones de extrema precariedad y articular acciones intersectoriales que contribuyan a la "seguridad epidémica, alimentaria y de otro tipo para contingentes de población cada vez más empobrecidos" (Seixas et al., 2021, p. 8).

10 Como estrategia para reducir la propagación de la pandemia, el trabajo de la APS también puede beneficiarse de su histórica y exitosa experiencia en la implementación del Programa Nacional de Inmunización. Esta dimensión es especialmente importante, tanto por la disponibilidad de diferentes vacunas contra el Covid-19 y sus diferentes intervalos entre dosis, como por un hecho que puede comprometer la protección de la vacunación en Brasil: hasta este momento, más de medio millón de personas que recibieron la primera dosis de Coronavac, la principal vacuna aplicada en el país, no han vuelto para recibir la segunda dosis, cuyo plazo debería ser de hasta 28 días después de la primera (Gamba \& Righetti, 2021).

11 La política central de expansión de la APS es la Estrategia de Salud de la Familia (ESF), cuyo proceso de trabajo involucra un equipo multiprofesional, compuesto por médicos, enfermeros, auxiliares de enfermería y agentes comunitarios de salud (ACS), categoría profesional que existe exclusivamente en el SUS. En marzo de 2020 se contabilizaban "44 mil equipos de ESF y 260 mil ACS distribuidos por los 5560 municipios del país" (Giovanella et al., 2021, pp. 14-15).

12 En este momento, pensamos que el trabajo de los ACS debe estar en relieve: su pilar es la relación estrecha con los usuarios y su esencialidad está en la educación en salud, acompañada del registro y producción de información para el diagnóstico territorial, mapeando las demandas y particularidades del territorio bajo su responsabilidad, principalmente a través de las visitas domiciliarias (VD) (Portaria no 2.436 , de 21 de setembro de 2017). Sin embargo, diversos estudios indican una histórica falta de reconocimiento de la labor de los ACS, corroborada por la subestimación de su papel en el enfrentamiento de la pandemia, generando, por ejemplo, una distribución desigual de los equipos de protección personal (EPP) en detrimento de esta categoría profesional (Nogueira et al., 2020). Es importante destacar que, aunque es evidente que las atribuciones de la APS son fundamentales para enfrentar la pandemia, su potencia no se limita a las prescripciones ya señaladas. Al tener como propósito el autocuidado en la salud, en el centro del trabajo de la APS está la relación de servicio, que se caracteriza por producir una transformación en las condiciones de actividad y en las disposiciones 
de acción del usuario/cliente (Zarifian, 2001). Frente el grave cuadro epidemiológico, el autocuidado debería también basarse en la solidaridad, en la expectativa de que sus efectos trasciendan la esfera individual y privada de los usuarios, produciendo repercusiones positivas en la vida comunitaria.

Al depender de la apropiación por parte del usuario/cliente de lo que se le ofrece, la producción de servicios se basa en la movilización de competencias profesionales para identificar y conocer la actividad del usuario/cliente, interpretar y comprender su punto de vista, movilizar recursos y actuar con pertinencia en las condiciones de ejecución (Zarifian, 2001). De esa manera, la producción del servicio extrapola el momento de la interacción y demanda la movilización de la actividad, individual y colectiva, en un contexto sociotécnico (Salini, Jaramillo, Goudeaux, \& Poizat, 2018).

En este sentido, se subraya un rasgo característico del trabajo en salud en la APS: está abierto a formas de vida parcialmente imprevistas de los usuarios/pacientes, lo que impide la total anticipación de las acciones de salud y exige un retroceso de las normas prescritas. En la APS, el trabajo en salud exige una postura mucho más comprensiva que prescriptiva, y las formas de alcanzar los objetivos y resultados dependen, en particular, de la iniciativa de los trabajadores.

Hay, pues, una invitación ineludible: interrogar el trabajo en salud en la APS a partir de la actividad movilizada por sus trabajadores. En medio de tantas lagunas sobre el Covid-19, dejarse interpelar por la actividad de trabajo significa dar visibilidad a los debates entre las normas precedentes y las tendencias de renormatización de los trabajadores (Schwartz, Adriano, \& Abderrahmane, 2008), buscando capitalizar los conocimientos y las elecciones hechas en los intentos de mitigar los efectos de salud y efectos sociales de la pandemia en el territorio brasileño, accediendo a eficacias potencialmente agenciables en los patrimonios colectivos.

Este encaminamiento también permite dar visibilidad al diálogo con los valores que circulan en los territorios, analizar las posibilidades e imposibilidades para el deslizamiento y afirmación en la dirección de los valores orientados al bien común, una cuestión de absoluta importancia en una sociedad tan marcada por la banalización de la violencia y la muerte como la brasileña. Se trata, por lo tanto, de ir más allá de la denuncia de las actuaciones gubernamentales en el gerenciamiento de la crisis sanitaria, que transitan, como mínimo, entre la omisión y la desorganización.

17 La interpelación del trabajo en salud desde el punto de vista de la actividad debe orientar la comprensión tanto de los recursos como de los obstáculos a la acción de los trabajadores, a fin de apoyar la provisión de condiciones objetivas favorables al desarrollo y a la consolidación de competencias y conocimientos tejidos en la adhesión de los territorios (Schwartz et al., 2008).

Por último, se han desperdiciado las potencialidades del trabajo de la APS. No obstante el escenario previo a la pandemia -marcado por la falta de inversiones, por el histórico desfinanciamiento del SUS y por el nuevo modelo de financiación de la APS (Daumas et al., 2020) -, sumado a la inexistencia de una coordinación nacional de la pandemia en el SUS, se probaron algunas iniciativas que invirtieron en la reorganización del trabajo en salud en la APS, como se muestra a continuación. 


\section{Reorganizaciones del trabajo en la APS durante la pandemia}

19 En Brasil, un encaminamiento común de la organización de la atención a la salud ha sido el cierre de las unidades y el redireccionamiento de los equipos para polos de atención y cuidado de los pacientes con síntomas respiratorios, generando la desarticulación de la asistencia sanitaria a los usuarios bajo su responsabilidad en los territorios.

Por otro lado, se han desarrollado alternativas de respuesta a la pandemia en la APS de forma integrada a la Vigilancia Epidemiológica, algunas destacadas por su potencialidad para producir un impacto positivo en la lucha contra el nuevo coronavirus. Así, y mediante exigencias de distanciamiento físico, se desarrollaron algunas experiencias de reorganización del trabajo pautadas por la telesalud, con prestación de servicios sanitarios a distancia e intermediados por las tecnologías de la información y la comunicación (TIC).

21 Por ejemplo, en la búsqueda de mantener la oferta de acciones de salud para la población como un todo, las Unidades de APS de las ciudades de Atalaia (AL), Río de Janeiro (RJ) y Vilhena (RO) reorientaron sus flujos y su proceso de trabajo integrando la continuidad de la atención a los pacientes con enfermedades crónicas a la atención de los casos sospechosos de Covid-19. Asociaron igualmente la telemonitorización y la vigilancia de los casos de Covid-19 en el territorio con la telemonitorización/ teleconsultas y las visitas a domicilio dirigidas a los usuarios en situación de vulnerabilidad (Favoreto, 2020).

22 En los municipios de Atalaia (AL) y Río de Janeiro (RJ), Favoreto (2020) también destacó la formación continua de todos los miembros del equipo de ESF y la movilización de la comunidad, con la participación de los líderes y organizaciones locales en las intervenciones de educación y promoción de la salud, mediante el uso de los medios sociales, como los grupos de WhatsApp y las videollamadas. En Vilhena (RO), en la región norte de Brasil, Riva, Moreira, Silva y Oliveira, (2020, p. 24) afirmaron que las acciones realizadas "redujeron drásticamente las formas de contagio de Covid-19 en las Unidades Básicas de Salud (UBS) o en contexto domiciliario", consolidando a la ESF.

En la ciudad de Londrina (PR), un equipo de la Residencia Multiprofesional en Salud de la Familia se comprometió con los equipos de salud de la UBS Padovani/Vista Bela -con énfasis en la actuación de los ACS- en la divulgación de información sobre formas de protección contra la contaminación, orientación a los usuarios en riesgo de Covid-19, nuevos modos de operación de la UBS y en la lucha contra la circulación de noticias falsas en las redes sociales (fake news).

Prepararon boletines informativos y los distribuyeron en grupos de WhatsApp/ Facebook de usuarios, como grupos de compra y venta de la región, grupo de información vecinal, grupo de Actividad Física de la UBS, grupos de las iglesias católica y evangélica, etc., poniéndose a disposición de los usuarios para aclarar sus dudas. También se creó un grupo de WhatsApp con los principales líderes comunitarios del territorio. A medida que se actualizaba la información, los estudios y las conclusiones sobre el Covid-19, se elaboraban y difundían nuevos informes. En colaboración con los usuarios interesados, crearon el proyecto "Cuídate, Vista Bela", que fue posible concretar gracias a los fondos donados por un proyecto social de una iglesia local. 
Fueron producidos y distribuidos 100 carteles con información relevante para la pandemia en tiendas, paradas de autobús, edificios y postes, además de la creación de canales de comunicación vía WhatsApp con la UBS.

Una experiencia similar se desarrolló en una UBS rural del interior de Pernambuco. Los profesionales de la salud también convirtieron la aplicación WhatsApp en un canal de comunicación con los usuarios para aclarar dudas sobre la pandemia, sobre los servicios disponibles en la unidad de salud y divulgar semanalmente un programa de radio sobre este tema (Cardona, Andrade, \& Caldas, 2020).

En el municipio de Coari, en Amazonas, se destacó la importancia de la APS con énfasis en la reorganización del trabajo de los ACS. En este estado, la principal forma de acceso a la atención sanitaria es mediante la APS, como es el caso de la ciudad de Coari, ubicada a 363 kilómetros de distancia de la capital Manaus y cuyo acceso es solamente posible por vía fluvial y aérea. En virtud de las barreras geográficas, la telesalud ha encontrado obstáculos como la "indisponibilidad y/o la baja calidad de los dispositivos, líneas telefónicas o el acceso a Internet en las unidades, bien como de los usuarios, la cuestión de la privacidad en los domicilios o incluso la capacidad de manipulación por parte de los pacientes" (Mata et al., 2020, p. 6). A pesar de estas dificultades, todas las unidades del municipio fueron equipadas con al menos un teléfono móvil con acceso a Internet, un recurso utilizado siempre que es posible.

Es en este contexto que el trabajo realizado por los ACS en los territorios tiene una importancia elevada. En las situaciones en que no es posible contactar remotamente con los usuarios, los ACS realizan visitas domiciliarias (sólo en casos de extrema necesidad) y la búsqueda activa de los usuarios en riesgo, respetando los protocolos sanitarios. Así, es importante situar que este protagonismo del trabajo de los ACS se desarrolla en un estado con uno de los peores indicadores de morbimortalidad por el nuevo coronavirus en Brasil (Mata et al., 2020), habiendo vivido, en enero de 2021, el episodio de colapso del sistema de salud, con la falta de oxígeno en los hospitales de la capital Manaus generando muertes y poniendo en riesgo a muchos pacientes (G1, 2021).

Aunque se identifica de forma más explícita e intensa el uso de las TIC durante la pandemia, cabe destacar que estas herramientas ya estaban reguladas por el Ministerio de Sanidad con anterioridad (Maciel et al., 2020). Aun así, en muchos casos su viabilidad en el trabajo en salud sólo fue posible con el uso de los propios dispositivos personales de los trabajadores, como atestiguan Bousquat et al. (2020, pp. 17-18) en su investigación: "Llama la atención la escasa disponibilidad de teléfonos móviles institucionales, ya que más del $70 \%$ de los profesionales que han respondido a la encuesta afirmaron utilizar su teléfono móvil personal para contactar con los usuarios".

29 La implantación de las TIC, principalmente en el trabajo de los ACS, una categoría con menos visibilidad social en la atención, debe tratarse con precaución. Es necesario monitorear los cambios efectivos provocados en las prácticas reales de trabajo, las exigencias generadas, las construcciones sociotécnicas que las materializan, sus efectos en la salud y las dimensiones sociales del trabajo (Baudin \& Nusshold, 2018).

Y, en general, las experiencias de reorganización del trabajo en la APS merecen una mejor comprensión, tanto en lo que respecta a su alcance para frenar la pandemia, como por los efectos en la salud de los trabajadores en los diferentes frentes de intervención. 


\section{El trabajo en salud en la APS y las reservas de alternativas}

31 Ante nuestra enorme tarea planetaria, muchos han señalado la importancia de extraer lecciones de esta crisis sanitaria. En lo que respecta al trabajo en salud en la APS, sus potencialidades no están contenidas sólo en sus atribuciones prescritas, sino sobre todo en la forma como son concretadas por sus trabajadores y en la capacidad de construir un patrimonio colectivo. Así, la indicación de aprendizajes en el debate público -como la valorización del SUS, el reconocimiento de la importancia del trabajo en equipo y la necesidad de avanzar en la cualificación de los trabajadores- son pistas de reservas de alternativas (Schwartz, 2011) que esperan ser visibilizadas y afirmadas.

Debe acentuarse la atención a las reservas de alternativas. Es alarmante que la existencia del SUS y la experiencia de la APS, ya consolidada en Brasil, no hayan conseguido producir las mejores respuestas en términos de prevención y control del Covid-19 (Giovanella et al., 2021). Entre los problemas en la respuesta de los sistemas de salud y de la APS, Giovanella et al. (2021) asocian "el predominio de los enfoques biomédicos, individualistas y asistenciales" (p. 21). A pesar de este análisis, se cuestiona: ¿la subestimación y la infrautilización de la APS también estarían relacionadas con las dificultades en construir y movilizar patrimonios en el SUS y la APS?

Bajo este ángulo, se considera relevante añadir otro aspecto: la propia invisibilidad de la actividad implicada en la intencionalidad de transformar las acciones de salud en la APS en una relación de servicio. El encuentro con los usuarios está atravesado por dimensiones parcialmente imprevisibles y relacionadas con su producción de normas de vida que evoca, de forma ampliada, la dimensión de gestión del trabajo. De este modo, el acceso a las reservas de alternativas producidas en el SUS y en la APS exige abordar el trabajo en salud desde el punto de vista de la actividad (Duraffourg, 2007/2010).

En este horizonte, se sugiere el reposicionamiento de la formación de los trabajadores, el aprendizaje continuo y la gestión de las unidades sanitarias a través de su instrucción desde el punto de vista de la actividad, es decir, en la dirección de la ergoformación y la ergogerenciamiento (Schwartz, 2011). Dichas acciones favorecerían la emergencia de las opciones y los valores presentes en el proceso de reelaboración de las normas precedentes para superar sus lagunas. Si "la salud empieza con un intento de rediseñar parcialmente el entorno en el que se vive, en función de sus propias normas" (Schwartz, 2011, p. 139), este reposicionamiento contribuye a que los trabajadores tengan, también ellos, su salud afirmada y fortalecida (Brito, 2017). 


\section{BIBLIOGRAFÍA}

Baudin, C., \& Nusshold, P. (2018). Editorial. Laboreal, 16(2). https://doi.org/10.4000/laboreal.16773

Bousquat, A., Nedel, F., Lima, J., Giovanella, L., Medina, M., Mendonça, M., ... \& Aquino, R. (2020). Desafios da atenção básica no enfrentamento da pandemia da Covid-19 no SUS (Relatório de Pesquisa/ 2020). Rio de Janeiro, Rede de Pesquisa em APS Abrasco. Recuperado de https://redeaps.org.br/ wp-content/uploads/2020/08/RelatorioDesafiosABCovid19SUS.pdf

Brito, J. (2017). Saúde: uma relação com o meio e os modos de vida. Laboreal, 13(1). https:// doi.org/10.4000/laboreal.2018

Cardona Jr., A., Andrade, C., \& Caldas, L. (2020). Educação em saúde: programa e canal de comunicação via WhatsApp da unidade básica de saúde do N6 para comunidade rural do sertão pernambucano. APS em Revista, 2(2). https://doi.org/10.14295/aps.v2i2.92

Conselho Federal de Enfermagem (2021a, 8 de janeiro). Brasil representa um terço das mortes de profissionais de enfermagem por Covid-19. Conselho Federal de Enfermagem. Disponível em http:// www.cofen.gov.br/brasil-responde-por-um-terco-das-mortes-de-profissionais-de-enfermagempor-Covid-19_84357.html

Conselho Federal de Enfermagem (2021b, 9 de março). Brasil perde ao menos um profissional de saúde a cada 19 horas para a Covid. Conselho Federal de Enfermagem. Disponível em http:// www.cofen.gov.br/brasil-perde-ao-menos-um-profissional-de-saude-a-cada-19-horas-para-aCovid_85778.html

Dantas, A. (2020). Coronavírus, o pedagogo da catástrofe: lições sobre o SUS e a relação entre público e privado. Trabalho, Educação e Saúde, 18(3). https://doi.org/10.1590/1981-7746-sol00281

Daumas, R., Silva, G., Tasca, R., Leite, I., Brasil, P., Greco, D., Grabois, V., \& Campos, G. (2020). O papel da atenção primária na rede de atenção à saúde no Brasil: limites e possibilidades no enfrentamento da COVID-19. Cadernos de Saúde Pública, 36(6). https://doi.org/ 10.1590/0102-311x00104120

Duraffourg, J. (2007/2010). O trabalho e o ponto de vista da atividade. In Y. Schwartz, \& L. Durrive (Eds.), Trabalho e Ergologia: conversas sobre a atividade humana (pp. 25-46). Niterói: EdUFF.

Fassa, A. C. (2021). "Temos que aproveitar esse momento de recrudescimento para avaliar onde estamos errando". Entrevista por Julia Neves. POLI: saúde, educação e trabalho, 13(75), 18-21. Disponível em http://www.epsjv.fiocruz.br/sites/default/files/poli_75_web_4.pdf

Favoreto, C. (2020). Atenção primária forte: elemento central no combate à pandemia de COVID-19. In C. Teixeira, C. Favoreto, D. Santos, L. Savassi, M. Guilam, M. Machado, \& M. Pinto (Eds.), COVID-19 e atenção primária: as experiências nos territórios (pp. 14-19). Recuperado de https:// profsaude-abrasco.fiocruz.br/sites/default/files/publicacoes/livro_-_Covid-19_e_aps.pdf

G1 (2021, 14 de janeiro). Covid-19: Manaus vive colapso com hospitais sem oxigênio, doentes levados a outros estados, cemitérios sem vagas e toque de recolher. G1, Amazonas. Disponível em https:// g1.globo.com/am/amazonas/noticia/2021/01/14/Covid-19-manaus-vive-colapso-com-hospitaissem-oxigenio-doentes-levados-a-outros-estados-cemiterios-sem-vagas-e-toque-derecolher.ghtml

Gamba, E., \& Righetti, S. (2021, 9 de abril). Mais de 500 mil pessoas que receberam a $1^{\text {a }}$ dose da vacina contra a Covid no Brasil não tomaram a $2^{\mathrm{a}}$. Folha de São Paulo. Recuperado de https:// 
www1.folha.uol.com.br/equilibrioesaude/2021/04/mais-de-500-mil-pessoas-que-receberama-1a-dose-da-vacina-contra-a-Covid-no-brasil-nao-tomaram-a-2a.shtml

Giovanella, L., Vega, R., Tejerina-Silva, H., Acosta-Ramirez, N., Parada-Lezcano, M., Ríos, G., ... \& Feo, O. (2021). ¿Es la atención primaria de salud integral parte de la respuesta a la pandemia de Covid-19 en Latinoamérica? Trabalho, Educação e Saúde, 19. https://doi.org/10.1590/1981-7746solo0310

Guimarães, C. (2020, 25 de março). A importância de um sistema de saúde público e universal no enfrentamento à epidemia. Fundação Oswaldo Cruz, Escola Politécnica de Saúde Joaquim Venâncio. Disponível em https://www.epsjv.fiocruz.br/noticias/reportagem/a-importancia-de-umsistema-de-saude-publico-e-universal-no-enfrentamento-a

Jackson, J. M., Assunção, A., Algranti, E., Garcia, E., Saito, C., \& Maeno, M. (2020). A saúde do trabalhador e o enfrentamento da COVID-19. Revista Brasileira de Saúde Ocupacional, 45(e14). https://doi.org/10.1590/2317-6369ed0000120

Maciel, F., Santos, H., Carneiro, R, Souza, E., Prado, N., \& Teixeira, C. (2020). Agente comunitário de saúde: reflexões sobre o processo de trabalho em saúde em tempos de pandemia de Covid-19. Ciência \& Saúde Coletiva, 25(2). https://doi.org/10.1590/1413-812320202510.2.28102020

Mata, M., Castro, D., Gomes, C., Macedo, J., Checchi, M., Gama, A., \& Souza, L. (2020). A experiência da reorganização da atenção primária à saúde - APS e trabalho dos agentes comunitários de saúde frente à COVID-19 em um município no interior do Amazonas. Journal of Management \& Primary Health Care, 12(40). https://doi.org/10.14295/jmphc.v12.1014

Medina, M., Giovanella, L., Bousquat, A., Mendonça, M., \& Aquino, R. (2020). Atenção primária à saúde em tempos de COVID-19: o que fazer? Cadernos de Saúde Pública, 36(8). https://doi.org/ 10.1590/0102-311x00149720

Nogueira, M., Borges, C., Lacerda, A., Fonseca, A., Vellasques, A. , ... \& Rego, S. (2020). $1^{\circ}$ Boletim da pesquisa Monitoramento da saúde dos ACS em tempos de Covid-19. Rio de Janeiro: Fiocruz.

Portaria nº 2.436, de 21 de setembro de 2017 (2017). Aprova a Política Nacional de Atenção Básica, estabelecendo a revisão de diretrizes para a organização da Atenção Básica, no âmbito do Sistema Único de Saúde. Recuperado de https://bvsms.saude.gov.br/bvs/saudelegis/gm/2017/ prt2436_22_09_2017.html

Riva, C., Moreira, K., Silva, E., \& Oliveira, J. (2020). Organizando fluxos no contexto da CoVID-19: vivências em uma equipe no norte do país. In C. P. Teixeira, C. Favoreto, D. Santos, L. Savassi, M. Guilam, M. Machado, \& M. Pinto (Eds.), COVID-19 e atenção primária: as experiências nos territórios (pp. 21-25). Mestrado Profissional em Saúde da Família - PROFSAÚDE.

Salini, D., Jaramillo, J., Goudeaux, A., \& Poizat, G. (2018). Profesiones de servicio y digitalización: implicaciones y sugerencias para la concepción de procesos de formación. Laboreal, 14(2). https:// doi.org/10.4000/laboreal.584

Schmitt, G. (2021, 26 de janeiro). Quase mil profissionais de saúde morreram por Covid-19 no Brasil. o Globo. Disponível em https://oglobo.globo.com/sociedade/quase-mil-profissionais-desaude-morreram-por-Covid-19-no-brasil-24853318

Schwartz, Y. (2011). Manifesto por um ergoengajamento. In P. F. Bendassolli, \& L. A. Soboll (Orgs.), Clínicas do trabalho: novas perspectivas para compreensão do trabalho na atualidade. São Paulo: Atlas. 
Schwartz, Y., Adriano, R., \& Abderrahmane, F. (2008). Revisitar a actividade humana para colocar as questões do desenvolvimento: projecto de uma sinergia franco-lusófona. Laboreal, 4(1). https:// doi.org/10.4000/laboreal.12192

Seixas, C., Merhy, E., Feuerwerker, L., Santo, T., Slomp, H., \& Cruz K. (2021). A crise como potência: os cuidados de proximidade e a epidemia pela Covid-19. Interface (Botucatu), 25(1). https://doi.org/10.1590/interface.200379

Teixeira, C., Soares, C., Souza, E., Lisboa, E., Pinto, I., Andrade, L., \& Espiridião, M. (2020). A saúde dos profissionais de saúde no enfrentamento da pandemia de Covid-19. Ciência \& Saúde Coletiva, 25(9), 3465-3474. https://doi.org/10.1590/1413-81232020259.19562020

Teixeira, M., Medina, M.., Costa, M., Barral-Netto, M., Carreiro, R., \& Aquino, R. (2020).

Reorganização da atenção primária à saúde para vigilância universal e contenção da COVID-19. Epidemiologia e Serviços de Saúde, 29(4), 1-5. https://doi.org/10.5123/s1679-49742020000400015

Zarifian, P. (2001). Objetivo competência: por uma nova lógica. São Paulo: Atlas.

\section{AUTORES}

\section{ANA CLÁUDIA BARBOSA DA SILVA-ROOSLI}

https://orcid.org/0000-0003-3795-9357

Departamento de Psicologia Social e Institucional da Universidade Estadual de Londrina (UEL), Rodovia Celso Garcia Cid, PR 445 Km 380, Campus Universitário, 86057-970, Londrina-PR, Brasil anaclaudia79@uel.br 